\title{
Estrés escolar y empatía en estudiantes de bachillerato practicantes de Mindfulness*
}

\author{
OLGA LUCÍA SIERRA** \\ origen.gdi@gmail.com \\ GUSTAVO URREGO ${ }^{* * * *}$ \\ origen.gdi@gmail.com \\ SANDRO MONTENEGRO ${ }^{* * * * *}$ \\ origen.gdi@gmail.com \\ CAMILO CASTILLO ${ }^{* * * * *}$ \\ origen.gdi@gmail.com
}

Recepción: 23 de octubre de 2014

Aprobación: 09 de febrero de 2015

Forma de citar este artículo: Sierra, 0. et al. (2015). Estrés escolar y empatía en estudiantes de bachillerato practicantes de Mindfulness. Cuadernos de Lingüística Hispánica, 26, 175-197, Tunja: Uptc.

Artículo resultado de una investigación, del programa Mindfulness, propuesta por la línea de Budismo.

** Ingeniera Química de la Universidad Nacional de Colombia. Co-Directora Centro de Meditación Budista Tibetana Yamantaka. Profesora de Budismo Tibetano en la Universidad del Rosario. Investigadora del Grupo Origen.

**** Psicólogo de la Universidad Nacional de Colombia. Psicoterapeuta Life Coach certificado AICP. Master life \& bussines coaching. Certificación internacional de Coaching. En AICP de España. Miembro del grupo de investigación Origen.

***** Filósofo de la Universidad Nacional de Colombia. Magíster en Filosofía contemporánea de la Universidad Central. Docente de cátedra de la Universidad Minuto de Dios. Docente del Distrito de Bogotá.

****** Psicólogo de la Fundación Universitaria Los Libertadores, Colombia. Líder del grupo de investigación Origen. 


\title{
Resumen
}

Este artículo expone el resultado de una investigación que contempló la práctica de Mindfulness dentro de un marco científico y pedagógico. Se seleccionó el estrés escolar y la empatía como variables para medir aplicando cuestionarios a dos grupos de adolescentes de la Institución Educativa Distrital "Juan Lozano Lozano" de la Localidad de Suba en Bogotá. Constituyó un estudio cuantitativo de carácter correlacional con un diseño cuasiexperimental de mediciones pre y post, con grupo control; el tratamiento fue la práctica meditativa durante dos meses. Para analizar la existencia de diferencias significativas entre las medidas pretest y postest, tanto en el grupo experimental como en el grupo control; se utilizó el $t$ de Student para muestras relacionadas. Se realizó un análisis entre el grupo experimental y el grupo control a partir de las diferencias antes y después de la intervención en ambos grupos, utilizando la prueba $t$ de Student para muestras independientes.

Palabras clave: Mindfulness, estrés escolar, empatía, rendimiento escolar.

\section{School stress and empathy in high school practitioners of Mindfulness}

\begin{abstract}
This article exposes the results of a research project on Mindfulness practice within a scientific and pedagogical frame. School stress and empathy were selected as variables to be measured through questionnaires in two teenage groups of Institución Educativa Distrital Juan Lozano Lozano in Suba locality of Bogotá. This constituted a correlational quantitative study, with a quasi-experimental design using a control group, and pre and post measurements. The treatment consisted in a two month practice of meditation. In order to analyze the existence of significant differences between the pre-test and post-test measurements, both in the experimental and control groups, the Student's $t$ was used for related samples. An analysis of the experimental and control groups was carried out based on the differences before and after the intervention in both groups, using the student's $t$ test for independent samples.
\end{abstract}

Keywords: Mindfulness, school stress, empathy, academic performance. 


\section{Stress scolaire et empathie chez les élèves des études secondaires, pratiquants du Mindfulness}

\section{Résumé}

Cet article expose le résultat d'une recherche qui a envisagée la pratique du Mindfulness dans un cadre scientifique et pédagogique. On a sélectionné le stress scolaire et l'empathie en tant que variables pour mesurer, en appliquant des questionnaires, aux groupes d'adolescents de l'Institution Éducative Distrital Juan Lozano Lozano de la Communauté de Suba à Bogotá. Ce travail a constitué une étude quantitative de caractère corrélationnel avec un design quasi expérimental de mesures pré et post, avec un groupe de contrôle; le traitement a été la pratique méditative pendant deux mois. Pour analyser l'existence de différences significatives entre les mesures prétest et posttest, tant dans le groupe expérimental, que dans le groupe de contrôle, on a utilisé le $t$ de Student pour des échantillons liés. On a fait une analyse entre le groupe expérimental et le groupe contrôle à partir des différences avant et après l'intervention de deux groupes, en utilisant le test $t$ de Student pour des échantillons indépendants.

Mots clés: Mindfulness, stress scolaire, empathie, rendement scolaire.

\section{Estresse escolar e empatia em estudantes do Ensino Médio praticantes de Mindfulness}

\section{Resumo}

Este artigo expõe o resultado de uma pesquisa que contemplou a prática de Mindfulness dentro de um marco científico e pedagógico. Selecionou-se o estresse escolar e a empatia como variáveis para medir aplicando questionários a dois grupos de adolescentes da Instituição Educativa Distrital Juan Lozano Lozano da Localidade de Suba em Bogotá. Constituiu um estudo quantitativo de caráter correlacional com um desenho quase experimental de medições pré e pós, com um grupo de controle; o tratamento foi a prática meditativa durante dois meses. Para analisar a existência de diferenças significativas entre as medidas pré-teste e pós-teste, tanto no grupo experimental como no grupo de controle, se utilizou o $t$ de Student para amostras relacionadas. Realizou-se uma análise entre o grupo experimental e o grupo de controle a partir das diferenças antes e depois da intervenção em ambos os grupos, utilizando a prova $t$ de Student para amostras independentes.

Palavras chave: Mindfulness, estresse escolar, empatia, rendimento escolar. 


\section{Introducción}

Mindfulness o meditación de atención plena, ha sido un tema de creciente interés para la ciencia occidental en los últimos años, los estudios neurocientíficos en alianza con sus principales practicantes: los budistas han dado cuenta del enriquecimiento mental y comportamental que puede llegar a tener quien lo practique. Es por ello que esta práctica oriental ha sido también aplicada e investigada en los ámbitos escolares, sobre todo en Europa y Norteamérica, donde se plantea como un componente importante dentro del proceso de formación a estudiantes de educación media.

Teniendo en cuenta que las relaciones sociales que logren los estudiantes en su contexto escolar es un factor importante para desarrollar habilidades emocionales y sociales que repercuten en el resto de la vida, es importante acotar que el repertorio de respuesta ante situaciones novedosas o estresantes puede llegar 0 no a contribuir al fortalecimiento de comportamientos asertivos, con gestión emociona que, a su vez, incluye el proceso sencillo, y complejo al tiempo, de enfocar la atención a las situaciones del presente considerando sus consecuencias tanto positivas como negativas.

El ejercicio de Mindfulness, que busca enfocar la atención, es un estado, no un proceso, donde hay una posición acrítica de auto-observación. Se observa las imágenes mentales, pero sin pensar; el sentimiento, sin implicarse en él; y de manera contemplativa y pasiva se observa cualquier contenido cognoscitivo, sensorial, emocional, fisiológico o conductual (Aguilar \& Musso, 2008).

Un método clave para el ejercicio de esta conciencia en las prácticas de meditación budista consiste en el desarrollo de la atención plena. Otro método más específico para el cultivo de ese control, consiste en cobrar una conciencia continua del proceso real de pensamiento, observando la aparición de la mente y los pensamientos en el mismo momento en que emergen (Ekman \& Gyatso, 2009, p. 94).

En este sentido, Mindfulness (atención o conciencia plena) puede considerarse, con toda probabilidad, el proceso más eficaz para el desarrollo de la atención, 
autoconocimiento y crecimiento personal (Solberg, et al., 2000). Teniendo en cuenta que, el estado de conciencia plena es distinto al de relajación o de sueño, ya que durante dicho período se produce un incremento del nivel de alerta en lugar de una reducción (Jevning, 1988; Jevning, Anand, Biedebach y Fernando, 1996).

De hecho, las técnicas de Mindfulness han sido confundidas de forma errónea con un tipo de relajación, pues los patrones EEG (Electroencefalográficos) han demostrado la aparición de efectos fisiológicos diferentes, ya que en el estado de conciencia plena la mente está a la vez disipada y atenta (Dunn, Hartigan y Mikulas, 1999). Según establece Campagne (2004), mientras que las técnicas de relajación emplean la voluntad y la concentración para alcanzar un estado de paliativo, las técnicas de Mindfulness también utilizan la voluntad y la concentración aunque no para aliviar, sino para mantenerse alerta y para perder toda noción de contenido del pensamiento, sin dejar de estar alerta ni perder claridad mental (Franco, Soriano, Justo, 2010).

En realidad, Mindfulness es, en sí misma, algo muy simple y familiar, algo que todos nosotros hemos experimentado en numerosas ocasiones de nuestra vida cotidiana. Cuando se es consciente de lo que se está haciendo, pensando, o nuestra mente se encuentra vagando sin orientación alguna, saltando de unas imágenes a otras, de unos a otros pensamientos. Mindfulness es una capacidad humana universal y básica, que consiste en la posibilidad de ser conscientes de los contenidos de la mente momento a momento. Es la práctica de la autoconciencia. El primer efecto es el desarrollo de la capacidad de concentración de la mente. El aumento de la concentración trae consigo la serenidad. Y el cultivo de la serenidad nos conduce a un aumento de la comprensión de la realidad (tanto externa como interna) y nos aproxima a percibir la realidad tal como es (Simón, 2008).

En cuanto a estudios de Mindfulness relacionados con la atención, según Bishop et al. (2004) las habilidades de Mindfulness están relacionadas con tres áreas de la atención: la sostenida, la selectiva y la capacidad para dirigir e intercambiar el foco de atención de una idea a otra. Lo cual corrobora la importancia que tiene el impacto del ejercicio contemplativo, dado que la atención es la primera fase de un proceso complejo para conformarse en la persona practicante comportamientos de empatía o enmarcados dentro de las habilidades sociales y emocionales (León, 2008).

En el terreno educativo existen posibilidades de aplicación de las técnicas derivadas de Mindfulness, aunque las experiencias que se están llevando a cabo son poco numerosas, encontrándose las diversas iniciativas promovidas desde Norteamérica. Una experiencia reciente y destacable es el "Mindfulness Education Program for Children" (Programa de Educación en Atención Plena para Niños) desarrollado por Lawlor, Fischer y Schonert-Reichl desde el año 2005 en Vancouver (Canadá). 
El estudio no solo tuvo el carácter de implementar Mindfulness como un programa educativo sino considerarlo desde un carácter investigativo para determinar la efectividad. Los primeros resultados fueron prometedores, encontrándose mejoras significativas en estudiantes desde el aspecto como el optimismo, la conducta social y el autoconcepto (Schonert-Reichl y Lawlor, 2010 citado por Arguís, Bolsas, et al. Hernández, Salvador, 2012, p. 34).

Sin embargo, tratamientos basados en la conciencia siguen aumentando su popularidad en todo el mundo. En los EE.UU., solamente los tratamientos basados en Mindfulness se aplican en más de 250 centros de salud (Jha et al., 2007). Las prácticas de Mindfulness fueron sustentadas originalmente en Oriente (budista) como meditación (por ejemplo, Baer, 2003). A través de la práctica de la meditación, la capacidad de dirigir la atención de una persona puede ser desarrollada. Uno de los objetivos principales de intervenciones basados en la conciencia es la percepción de momento a momento de los hechos, con plena conciencia, sin distorsión de los pensamientos asociados del observador (Kabat-Zinn et al., 1985). El entrenamiento en Mindfulness se diferencia de las terapias de comportamiento convencionales (cognitivo), en la medida en que se centran en dificultades universales en lugar de en los problemas específicos (Bögels et al.,2008; Jiménez, 2011).

En cuanto a estudios realizados en el contexto escolar, Beauchemin, Hutchins y Patterson (2008) realizaron uno sobre las técnicas de Mindfulness en un colegio de Madrid, España. El estudio contó con un pre y post test, sin un control en el diseño de intervención. Con un total de 34 estudiantes voluntarios, con dificultades de aprendizaje, en edades entre 13 a 18 años, en un colegio especializado. Los profesores dirigían 5 a 10 minutos de meditación al inicio de cada jornada, diariamente durante 5 semanas. Mientras que los estudiantes que no participaron se encontraban en actividades no disruptivas dentro del aula. Los docentes no tenían experiencia en Mindfulness, a excepción de un entrenamiento previo a la intervención de 2 horas y 45. La autoevaluación de ansiedad y habilidades sociales, así como la evaluación por parte de los docentes sobre las habilidades sociales y los logros académicos, mostraron una diferencia significativa entre las evaluaciones pre y post test (Alajmo \& Jiménez, 2013).

En el país Vasco hay otra investigación donde se implementó Mindfulness en contexto escolar titulada "Aprendizaje y práctica de la conciencia plena en el contexto escolar", la población fue estudiantes sudamericanos que se encontraban cursando Primer Curso de Bachillerato, de los cuales cuarenta y nueve formaron parte de la investigación. 
El programa de intervención aplicado tuvo una duración de 10 semanas (segundo trimestre del curso académico), con una periodicidad de una sesión semanal de 1'30 de duración cada una de ellas. El programa de intervención aplicado en el grupo experimental consistió en el aprendizaje y práctica diaria, durante 30 minutos, de una técnica de Mindfulness para el desarrollo de la conciencia plena (Franco, 2007, 2009), cuyo objetivo principal no consiste en tratar de controlar los pensamientos ni modificarlos o cambiarlos por otros, sino todo lo contrario, dejarlos libres aceptando cualquier pensamiento que pueda aparecer 0 surgir de forma espontánea, con el fin de apaciguar la generación de pensamientos conceptuales (Franco, Soriano \& Justo, 2010, p. 4).

La metodología de la investigación referida tuvo dos grupos (control y experimental), se implementaron medidas pretest y postest, tomando como variables la autoestima y el rendimiento académico, tanto en el grupo experimental como en el grupo control; se utilizó la prueba $t$ de Student para muestras relacionadas. Los resultados confirmaron la hipótesis que señaló que el programa psicoeducativo de Mindfulness, para el desarrollo de la atención o conciencia plena, produce mejoras significativas en el grupo experimental en comparación con el grupo control, en las variables rendimiento académico y auto concepto (Franco, Soriano \& Justo, 2010).

Una revisión de literatura sobre Mindfulness y temas relacionados con las prácticas contemplativas, elaborado por Sánchez (2011) encontró:

treinta y ocho estudios científicos: 31 con metodología y análisis cuantitativos, 4 estudios piloto cualitativos, un estudio mixto cuantitativo cualitativo, un único estudio cualitativo, un meta-análisis correspondiente a una tesis de doctorado de la Universidad de Harvard que incluye 5 estudios cuasi experimentales; adicionalmente se presentan aportes de 7 revisiones de literatura que también incluyen sistematizaciones de estudios científicos, así como 5 textos clásicos o sobre modalidades de meditación complementarias (Sánchez, 2011, p. 225).

Las principales bases de datos consultadas fueron: Bicimohure, PubMed, Oxford Journals, EbscoHost y todas las del área de interés (Sport Discuss, PsyArticles), Human Kinetics. Asimismo, se incluye un meta-análisis de la Universidad de Harvard del año 2008 (Sánchez, 2011, p. 225).

En el contexto colombiano, aunque ha habido acercamientos entre el campo escolar y las prácticas contemplativas, falta evidencia de cómo esta práctica puede lograr cambios en el comportamiento de los estudiantes, sobre todo en la educación básica, invadida de problemáticas comportamentales como el estrés, entre otros, que pueden afectar la capacidad empática de los niños, niñas y adolescentes. 


\section{El estrés en el contexto escolar}

En cuanto a las variables que pueden afectar a los niños y niñas en su rendimiento en la institución educativa está el estrés escolar, el cual se presenta con mayor complejidad en el proceso formativo de un niño, niña o adolescente, ya que hay factores que precipitan sus componentes y pueden llevar a reacciones que desencadenan comportamientos disfuncionales difíciles de abordar.

Teniendo en cuenta que la exposición a situaciones de estrés no es en sí misma algo "malo" o que necesariamente conlleve efectos negativos, sólo cuando las respuestas -como consecuencia de innumerables estímulos o situaciones ambientales- son excesivamente intensas, frecuentes y duraderas pueden producir una variedad de trastornos fisiológicos en el organismo, situaciones que se conceptualizan en el término: estrés crónico (Arden, 2002; Rodríguez, Roque y Molerio, 2002).

El aspecto central que diferencia el estrés crónico del estrés basado en sucesos estresantes (por ejemplo, sucesos vitales y contrariedades) es el fenómeno de la continuidad. Los estresores crónicos suelen poseer un curso continuo, resultando improbable que pueda conceptuarse en términos de suceso. Los estresores crónicos son, típicamente, abiertos en el tiempo (no tienen un final claro). El comienzo de un estresor crónico es gradual, el curso es de larga duración y continuo (no se trata de una sucesión de eventos), y el final es problemático y frecuentemente impredecible (Sandin, 2002, p. 150).

En un estudio realizado en noventa escuelas de Norteamérica se encontraron las diferentes respuestas que tienen los adolescentes ante los síntomas de estrés crónico.

El estudio, basado en un cuestionario cuidadosamente construido, fue diseñado para reunir información sobre la frecuencia y grado cualitativo de varias formas de estrés, en grupos de adolescentes de edades comprendidas entre los 12-17 años. La primera medición tuvo lugar en 1986 e incluyó un total de aproximadamente 90 escuelas con 1.700 estudiantes de ambos sexos. Para conseguir información exacta a largo plazo de su desarrollo, el estudio fue repetido en 1987 y 1988 -adolescencia- (Buela-Casal, Fernández-Ríos, Carrasco, 1997).

Los resultados del estudio confirmaron que, bajo ciertas circunstancias, los adolescentes reaccionan a síntomas de estrés a constelaciones de riesgo en condiciones de vida. Además, se encontraron diferencias en las condiciones previas que conducen a enfermedades psicosomáticas y otras alteraciones de salud, perturbaciones psicológicas y emocionales, consumo de droga y delincuencia. Los "factores de riesgo" más importantes que llevaron al síntoma de estrés fueron: a) Padres muy exigentes en las expectativas 
de rendimientos en la escuela. b) Fuerte estrés subjetivo debido a demandas escolares. c) Fracaso escolar. d) Dificultades para alcanzar el reconocimiento e integración en el grupo de iguales, y e) Desventajas en los materiales en comparación con amigos e iguales. La ocurrencia de uno o más de estos factores de riesgo se correlaciona con una alta probabilidad de que surjan varios síntomas de estrés. Los resultados revelaron que: los síntomas de estrés dependen particularmente de la percepción subjetiva de estrés en el rendimiento escolar y del fracaso escolar objetivo.

La conducta social desviada y la delincuencia correlacionan fuertemente con la integración en grupos de iguales desviados. Este último hecho está conectado con el rendimiento escolar y la integración en un grupo de iguales apropiado. Por otro lado, el fracaso escolar y la integración en pandillas no apropiadas presentan también una alta correlación con el consumo de drogas.

Una reducida autoestima, así como la presencia de estrés psicosomático y emocional, es particularmente evidente cuando los niveles subjetivos de estrés son altos. "El estudio indica claramente que los adolescentes que viven inmersos en condiciones problemáticas y llenas de conflictos experimentan frecuentemente síntomas físicos, psicológicos y sociales de desarrollo desviado durante la adolescencia (Buela-Casal, Fernández-Ríos, Carrasco, 1997, p. 105).

Por lo tanto, hay evidencia de que el estrés en el contexto escolar surge de varias fuentes estresoras, pero la que más relevancia tiene es de carácter endógena, es decir las que provienen de la formación impartida por los padres, las relaciones entre padres e hijos, que en algunos casos, al contrario de formar al adolescente, le refuerzan conductas agresivas o pasivas en un contexto escolar donde se exigen habilidades sociales.

Una situación no es estresora por su naturaleza propia, ni por sí sola es causa suficiente para producir enfermedades. El desarrollo del estrés requiere de la presencia de otras condiciones, tales como tejidos vulnerables o estrategias de control ineficaces en la adolescencia (Buela-Casal, Fernández-Ríos, Carrasco, 1997, p. 106).

Witkin (2002), citado por Forero, López y Pardo (2004), realizó una investigación acerca del estrés infantil y encontró que la escuela puede ser un estresor importante en términos de competitividad, no solo por las calificaciones de los estudiantes, sino también por las rivalidades que hay entre compañeros, participación en clase, realización de exámenes, desarrollo de tareas, aceptación del grupo, miedo al fracaso y decepciones de los padres, entre otros (Martínez \& Díaz, 2007). 
En la misma línea, Dumont y otros (2003) realizaron un estudio en el que midieron el grado de estrés antes de un examen en adolescentes de Grado Décimo en Canadá. Dichos investigadores concluyeron que el 58\% de los estudiantes de una muestra de 374 presentaban lo que ellos llamaron "ansiedad antes de un examen".

Por su parte, Robinson y otros (1995) estudiaron el momento de transición al ingresar al bachillerato hasta undécimo grado, de una muestra de 371 estudiantes. Hallaron que hay una conexión significativa entre el estrés, el estilo atribucional y la valía personal al momento de presentarse una situación estresante (Martínez \& Díaz, 2007, p. 14).

En el contexto educativo colombiano son varios factores los que pueden llevar a desencadenar estrés escolar, como la misma convivencia con sus iguales y las demandas de los docentes. Sin embargo, el hecho de que existan estos detonantes, no quiere decir que siempre se presente estrés crónico, ya que en gran medida depende de los recursos de los niños, niñas y adolescentes dados en el hogar, lo cual suele confundirse con evasiones de parte del estudiante frente a sus deberes.

Desde luego, es importante tener en cuenta, para diferenciar el estrés escolar de otro tipo de situación, que este fenómeno que afecta a los estudiantes

Se define como el malestar que el estudiante presenta debido a factores físicos, emocionales, ya sea de carácter interrelacional o intrarrelacional, 0 ambientales que pueden ejercer una presión significativa en la competencia individual para afrontar el contexto escolar en rendimiento académico, habilidad metacognitiva para resolver problemas, pérdida de un ser querido, presentación de exámenes, relación con los compañeros y educadores, búsqueda de reconocimiento e identidad, habilidad para relacionar el componente teórico con la realidad específica abordada (Martínez \& Díaz, 2007, p. 14).

Por otro lado, Barraza (2005) hace una clasificación más detallada en cuanto a los fenómenos de estrés en el contexto escolar; considera que el estrés académico es aquel que los estudiantes padecen a consecuencia de exigencias endógenas y exógenas que impactan su desempeño en el ámbito escolar; señala que difiere de Polo, Hernández y Poza (1996), quienes argumentan que el Estrés Académico puede afectar de igual forma a docentes y estudiantes, pues el estrés de los docentes es laboral u ocupacional.

Según lo mencionado, el entorno puede generar estrés escolar cuando no existen condiciones y normas adecuadas que permitan un sano desarrollo de la socialización, que propicie comunicación entre los estudiantes con los profesores, sus padres y familiares, con la sociedad y las relaciones ambientales. El estrés escolar es un fenómeno presente y actuante. 
Es así como los estudiantes pueden padecer de estrés a lo largo del año académico, desde el ingreso a la institución educativa que marca la adaptación, las relaciones entre sí y con sus educadores, el proceso de tareas, las evaluaciones, el cansancio cognitivo, la frecuente indisciplina -indicador de algún tipo de malestar-, el incremento de la violencia en la escuela, el afán por culminar un ciclo académico más, son factores que resaltan (Martínez \& Díaz, 2007).

Ante todo, el objeto de toda medida de intervención es estimular y fortalecer los recursos individuales y sociales que deben ser utilizados con una situación difícil o con estresores duraderos. En lo que respecta a los recursos individuales, el énfasis radica en la promoción de competencias; en lo referente a los "recursos sociales", el énfasis se dirige a la promoción de redes de apoyo. Las medidas de intervención se deben dirigir hacia los polos de esta relación (Buela-Casal, Fernández-Ríos, Carrasco, 1997, p. 106).

\section{La empatía como componente importante de la inteligencia emocional}

Dentro de estos recursos sociales se encuentra un repertorio de herramientas de habilidades sociales y uno de sus componentes importantes es la capacidad de sentir a la otra persona en su pensamiento y emoción, gracias al equipamiento neuronal que posee el ser humano y más exactamente las neuronas espejo, podemos sentir lo que siente la otra persona.

En humanos, la evidencia de representaciones neuronales compartidas entre uno mismo y los otros se describió en primer lugar en el campo de la acción (Rizzolatti, Fogassi, Gallese, 2001) y la emoción (Carr, et al., 2003; Wicker, et al., 2003). Más recientemente, la investigación ha mostrado el papel de las representaciones compartidas en los dominios del procesamiento del dolor (Botvinick, et al., 2005; Morrison, Peelen, Downing, 2007) y del tacto (Avenanti, et al., 2006; Keysers, et al., 2004). Las neuronas espejo de las áreas promotoras, que se creía que estaban únicamente implicadas en el reconocimiento de una acción determinada, están también involucradas en la comprensión de la conducta de los otros (Lacoboni, et al., 1999; Tettamanti, et al. 2005). En este sentido, entender una intencionalidad es inferir un nuevo propósito que está por llegar, proceso que el sistema motor realiza de forma automática (Lacoboni, et al., 2005) (Moya-Albiol, Herrero, Bernal, 2010).

Eisenberg (2000) define la empatía como una respuesta emocional que procede de la comprensión del estado o situación de otra persona y es similar a lo que la otra persona está sintiendo. Por lo tanto, la respuesta empática incluye la capacidad para comprender al otro y ponerse en su lugar, a partir de lo que se observa, de la información 
verbal o de información accesible desde la memoria (toma de perspectiva) y además la reacción afectiva de compartir su estado emocional, que puede producir tristeza, malestar 0 ansiedad (Richaud, 2008).

Pero es precisamente la empatía, el componente hendido en la dinámica social de los adolescentes cuando hay conflictos o respuestas de estrés, dado que sobrepasa sobre este una carga emocional fuerte que enceguece al sujeto y lo impulsa a reacciones sin un procesamiento de la situación o de la persona que tiene al frente, perdiendo la capacidad empática.

Las investigaciones sobre empatía han demostrado que esta es una variable que interviene en las relaciones interpersonales y grupales, facilitando u obstaculizando las relaciones armoniosas con los demás. De Paul (2004) y Feshbach (1982) desarrollaron un modelo explicativo de la agresión en la que se considera la existencia de déficits de empatía en los sujetos y que la experiencia empática puede influir en la inhibición de los comportamientos agresivos, la atribución de responsabilidades y la ansiedad generada (García, et al, 2011).

Si la intervención solo se concentra en los esfuerzos del individuo por afrontar la tensión y evitar de este modo el contacto con las bases sociales de ese esfuerzo, resultará inefectiva 0 , a los más, producirá una sustitución del síntoma. El adolescente que bebe alcohol o fuma para aliviar el estrés, reconoce que tales conductas son perjudiciales para la salud; por tanto, puede cambiar su mecanismo de conflicto, y llegará a ser agresivo 0 depresivo, adquirir hábitos alimenticios malsanos o buscar refugio en otras conductas que perjudicarán su salud a largo plazo (Buela-Casal, et al., 1997).

Por tal motivo, las iniciativas y acciones preventivas frente a las problemáticas de los adolescentes en las instituciones educativas, tiene una tendencia de mejor repuesta al realizarse a través de proyectos que aborden las habilidades para la vida, donde se desprenden la inteligencia emocional y variables tan importantes como la empatía. Adicionalmente, es de gran interés general, de parte de la nueva pedagogía, introducir parámetros diferentes para generar mejores resultados, es de esta manera como han surgido iniciativas de proyectos de habilidades para la vida junto con Mindfulness.

En consideración a la importancia de iniciativas que rindan frutos de proyectos de prevención en las instituciones educativas, donde cobra importancia Mindfulness en procesos de gestión emocional, para prevenir, incluso, el estrés crónico escolar y fortalecer la empatía, se planteó la siguiente pregunta de investigación: ¿Qué relación tiene el estrés escolar con la empatía en un grupo de estudiantes de grado sexto del IED "Juan Lozano Lozano" de Bogotá, que en el término de dos meses practican Mindfulness? 


\section{Metodología}

La investigación fue de corte cuantitativo; por tal motivo, se hizo uso de la lógica deductiva, que parte de la búsqueda teórica, derivándose de allí las hipótesis, las cuales se someten a prueba teniendo en cuenta las diferentes variables. La recolección de información respecto de las variables proporcionaron datos suficientes para sintetizar algunas conclusiones que se basan en el cumplimiento de unos principios metodológicos, que exigen un desarrollo de estándares de diseños estrictos; de manera que se facilitó establecer, formular, fortalecer y revisar la hipótesis de trabajo, mediante métodos matemáticos que relacionan dichas variables (Sampieri, Fernández y Baptista, 2006).

El diseño de la investigación fue cuasiexperimental, ya que se manipuló deliberadamente una variable independiente para observar el efecto y relación con una o más variables dependientes. Adicionalmente, en los diseños cuasiexperimentales los sujetos no se asignan al azar a los grupos ni se emparejan, sino que dichos grupos ya están formados antes del experimento: son grupos intactos (Sampieri, Fernández y Baptista, 2006, p. 203).

Para complementar el proceso investigativo y tener una base sólida del diseño discriminando los efectos de la variable independiente, se implementó un diseño con preprueba-posprueba y grupo de control, el cual "incorpora la administración de pruebas a los grupos que componen el experimento, $[\ldots]$ un grupo recibe el tratamiento experimental y otro no (es el grupo control); por último, se les administra, también simultáneamente, una posprueba" (Sampieri, Fernández y Baptista, 2006, p. 193).

Se seleccionó una muestra compuesta por dos grupos de grados sextos, del IED "Juan Lozano y Lozano", de un total de cuarenta adolescentes de cada grupo, con edades entre los 12 y 14 años, tanto adolescentes hombres como mujeres. La institución educativa fue quien dio el consentimiento informado para permitir el estudio. Finalmente, se seleccionó una muestra poblacional no probabilística de 44 adolescentes.

La muestra no probabilística considera que los elementos no dependen de la probabilidad, sino de causas relacionadas con las características de la investigación o de quien hace la muestra. Aquí el procedimiento no es mecánico, ni con base en fórmulas de probabilidad, sino que dependen del proceso de una toma de decisiones de una persona o de un grupo de personas y, desde luego, las muestras seleccionadas obedecen a otros criterios de investigación (Sampieri, Fernández y Baptista, 2006, p. 241).

Teniendo en cuenta que el tipo de investigación fue correlacional y hay comparación de grupos, las hipótesis no direccionales plantean las predicciones del resultado de la investigación: 
- En dos meses de práctica de Mindfulness disminuye el estrés escolar y aumenta la capacidad empática en los estudiantes del grupo experimental.

- En dos meses de práctica de Mindfulness no disminuye el estrés escolar ni aumenta la capacidad empática en los estudiantes del grupo experimental.

\subsection{Instrumentos}

Los instrumentos seleccionados para la investigación permitieron identificar la modificación que asimilaron los estudiantes del experimento dada la práctica del Mindfulness, en las variables dependientes: estrés escolar y empatía; por lo tanto, se tuvo en cuenta una línea base (pre test) y posterior a los meses de práctica de la meditación se evalúa los cambios (postest).

Para la medición del estrés escolar, se utilizó el instrumento construido y validado por González, Lemus, \& Leal (2002) con once ítems. Los seis primeros ítems indican el tipo actitudinal y los restantes indican el carácter comportamental.

El instrumento consta de dos partes (A y B). La primera utiliza la técnica Likert (ítems 1 a 6) y la segunda, ítems de múltiple respuesta (ítems 7 a 11). Las preguntas 1 y 11 evaluaron la relación entre las variables de estrés con respecto al rendimiento académico que los estudiantes presentan. Las preguntas 2 y 9 evaluaron las variables de estrés, familia e institución educativa, recalcando en cómo opera el estudiante las relaciones familiares cuando se trata de la institución. Con las preguntas 3 y 8 se buscó valorar la variable relacionada con el área social dentro del contexto escolar y cómo enfrenta el estudiante las situaciones que, en algún momento, le generan temor o ansiedad dentro de dicho contexto. Las preguntas 4 y 7 estuvieron orientadas a conocer cómo el estudiante enfrenta la desmotivación hacia algunas materias y la manera cómo afronta situaciones en las que, en determinado momento, pueda sentirse nervioso o incómodo frente al grupo. Las preguntas 5 y 6 evaluaron la comprensión de lo explicado en el aula y la dificultad de concentración. La pregunta 10 pretendió evaluar la respuesta física que experimentan los estudiantes en el momento en que se encuentran nerviosos o preocupados (Martínez y Díaz, 2007).

En segundo lugar, se utilizó la Escala de empatía del cuestionario de conducta prosocial de Martorell, González y Calvo (1998) validada por Rey (2003) para la población colombiana. Escala que está dirigida a preadolescentes y adolescentes de los dos sexos. Con un total de 15 ítems, que se contestan a través de una escala tipo Likert de cuatro opciones, permitiendo obtener una puntuación mínima de 15 puntos y una máxima de 60 , donde se considera que son altas las puntuaciones que van desde 47 hacia arriba, media entre 32 y 40 y baja por debajo de 31 . Tiene una confiabilidad de 0.78 , con un nivel de 
significancia superior al 0.001 para la correlación ítems puntuación total de la escala (Rey, 2003).

\subsection{Procedimiento}

Fase 1. Selección de la muestra, según determinaron las directivas de las instituciones educativas, para mediar con las sesiones de Mindfulness, procedimiento que no fue mecánico, ni se fundamentó en fórmulas probabilísticas, fue tras un proceso de toma de decisiones (Sampieri, Fernández y Baptista, 2006).

Fase 2. Aplicación de pretest de las dos pruebas (estrés escolar y empatía), que se implementa a los grupos experimentales y de control en la institución educativa.

Fase 3. Aplicación: el programa de Mindfulness se aplicó, durante dos meses. La duración de cada sesión fue de aproximadamente 10 minutos.

Fase 4. Aplicación de postest de las dos pruebas (estrés escolar y empatía), que se implementa a los grupos experimentales y de control en la institución educativa.

Fase 5. Análisis de resultados: posterior a la tabulación de los resultados dados por los instrumentos aplicados.

Fase 6. Conclusiones e impacto del programa de Mindfulness, entrega de resultados a la institución educativa.

\section{Resultados}

Para analizar la existencia de diferencias estadísticamente significativas en las medidas pretest y postest entre ambos grupos, se utilizó la prueba $t$ de Student para muestras independientes (véase Tabla 1). La Tabla 1 muestra la no existencia de diferencias estadísticamente significativas entre ambos grupos en las medidas pretest en ninguna de las variables del estudio. Es decir, no existían diferencias entre los grupos en las variables analizadas antes de la intervención. 
Tabla 1. Medias (M) y desviaciones típicas (DT) pretest y postest correspondientes a los grupos control y experimental

\begin{tabular}{cccccccccc}
\hline & \multicolumn{3}{c}{ Pretest } & \multicolumn{5}{c}{ Postest } \\
& $\begin{array}{c}\text { Grupo } \\
\text { Experimental } \\
\text { M }\end{array}$ & DT & Grupo Control & \multicolumn{2}{c}{$\begin{array}{c}\text { Grupo } \\
\text { Experimental }\end{array}$} & \multicolumn{2}{c}{ Grupo Control } \\
& M & DT & M & DT & M & DT \\
\hline $\begin{array}{c}\text { Estrés escolar } \\
\text { Parte A }\end{array}$ & 22.8 & 2.97 & 26.6 & 2.47 & 22.3 & 2.29 & 23.2 & 3.07 \\
$\begin{array}{c}\text { Estrés escolar } \\
\text { Parte B }\end{array}$ & 12.5 & 2.08 & 14.6 & 2.99 & 12.4 & 1.93 & 13.3 & 1.93 \\
Empatía & 17.7 & 2.76 & 18.5 & 2.83 & 16.6 & 2.40 & 22.8 & 1.83 \\
\hline
\end{tabular}

Para comprobar si hubo cambios significativos en el grupo experimental respecto del grupo control, una vez finalizada la práctica de Mindfulness, se realizó un análisis de las diferencias postest entre las medidas del grupo experimental y del grupo control, y se obtuvo la aparición de diferencias significativas entre ambos grupos en las variables analizadas. Es decir, se produjeron diferencias significativas tanto en la variable de estrés escolar, parte A $(\mathrm{t}=4.37 ; \mathrm{p}=0.001)$, estrés escolar, parte $\mathrm{B}(\mathrm{t}=4.22 ; \mathrm{p}=0.001)$ y Empatía $(\mathrm{t}=1.07 ; \mathrm{p}=0.320)($ Ver Tabla 2$)$.

Tabla 2. Prueba $t$ de Student para muestras independientes de las diferencias pretest y postest entre el grupo control y experimental

\begin{tabular}{lcccc}
\hline & \multicolumn{2}{c}{ Pretest } & Postest \\
& $\mathbf{t}$ & $\mathbf{P}$ & $\mathbf{t}$ & $\mathbf{p}$ \\
Estrés escolar Parte A & 4.37 & 0.001 & 0.95 & 0.345 \\
Estrés escolar Parte B & 4.22 & 0.001 & 1.65 & 0.107 \\
Empatía & 1.07 & 0.320 & 3.18 & 0.003 \\
\hline
\end{tabular}

Para analizar si las posibles diferencias estadísticamente significativas entre las puntuaciones postest-pretest en el grupo experimental, se realizó un análisis estadístico mediante la prueba $t$ de Student para muestras relacionadas, se encontró la existencia de diferencias entre las puntuaciones postest-pretest del grupo experimental en todas las variables analizadas. 
De este modo, se produjeron diferencias tanto en la variable Estrés escolar parte A $(\mathrm{t}=4.75 ; \mathrm{p}=0.001)$, Estrés escolar parte $\mathrm{B}(\mathrm{t}=3.29 ; \mathrm{p}<0.005)$ y Empatía $(\mathrm{t}=8.07 ; \mathrm{p}<.001)$ (Ver Tabla 2).

Para considerar diferencias significativas entre las puntuaciones postest-pretest en el grupo control, se realizó un análisis estadístico mediante la prueba $t$ de Student para muestras relacionadas, y se encontró que no aparecían diferencias significativas entre ambas puntuaciones postest-pretest en todas las variables analizadas para dicho grupo (Ver Tabla 3).

Tabla 3. Prueba $t$ de Student para muestras relacionadas de las diferencias pretest y postest entre el grupo control y experimental

\begin{tabular}{lcccc}
\hline & \multicolumn{2}{c}{ Pretest } & \multicolumn{3}{c}{ Postest } \\
& $\mathbf{t}$ & $\mathbf{p}$ & $\mathbf{t}$ & $\mathbf{p}$ \\
Estrés escolar Parte A & 0.97 & 0.370 & 4.74 & 0.001 \\
Estrés escolar Parte B & 0.89 & 0.383 & 3.29 & 0.003 \\
Empatía & 1.17 & 0.105 & 8.07 & 0.000 \\
\hline
\end{tabular}

\section{Discusión}

Al ser analizados los resultados, se puede concluir que se confirma la hipótesis de investigación que refería la posibilidad de disminución del estrés escolar y el aumento de la empatía en los dos meses de práctica de Mindfulness, lo que significa que la implementación de las sesiones para el desarrollo de un equilibrio emocional, produjo mejoras en el grupo experimental en comparación con el grupo control en las variables estrés escolar y empatía.

Al realizar el análisis estadístico de las diferencias entre las puntuaciones postestpretest en el grupo experimental, se obtuvo diferencias en las variables dependientes (estrés escolar y empatía), observándose un incremento de las puntuaciones medias postest en empatía y un decremento en el estrés escolar en comparación con las puntuaciones medias pretest. Por el contrario, no hubo diferencias en el grupo control entre las puntuaciones medias postest y pretest en ninguna de las variables dependientes.

De esta manera, se puede concluir que hay una relación entre el estrés escolar y la empatía, puesto que en la medida que haya menor estrés escolar hay mayor empatía o se presta mejor el grupo para una convivencia con más capacidad de comprensión de 
emociones entre estudiantes. De igual forma, debe tenerse en cuenta que el tiempo de implementación de las sesiones de Mindfulness, puede ser un tiempo considerable para que se fortalezca la socialización entre los estudiantes.

Por lo tanto, los practicantes novatos de Mindfulness pueden llegar al punto de comprender que los pensamientos surgen y desaparecen continuamente, sometidos a un continuo flujo. Presentes, abiertos y equilibrados frente a cualquier fenómeno o proceso mental o emocional que acontezca. En este sentido, mediante la práctica de la conciencia plena se llega a un conocimiento mejor, no como un ideal, sino como realmente se es, sin tener la necesidad de cambiar o modificar nada para sentirnos bien consigo mismo (KabatZinn, 1990, 2005).

Con la práctica de Mindfulness se acepta la situación tal cual es, se liberan sentimientos que están influenciados por pensamientos y emociones de determinada situación, lo que contribuye a una imagen de sí mismo segura y estable (Holden, 2006).

Adicionalmente, se infiere que la disminución en los niveles de estrés escolar obtenido en el grupo experimental, pudo estar influido por el hecho de haber mejorado sus niveles de atención y concentración por la práctica de Mindfulness. Uno de los principales beneficios que se obtiene con la práctica continuada de Mindfulness es la de cesar el ritmo frenético y caótico de pensamientos que surgen de forma, puesto que ese constante fluir de pensamientos incontrolados distrae y dificulta la capacidad de atención y concentración (Austin, 1998).

Es importante señalar que, sin embargo el proceso de las sesiones no tuvo una continuidad diaria, puesto que se presentaban eventos de parte de la institución educativa o inconvenientes por parte de los guías del ejercicio de Mindfulness.

En futuras investigaciones se debe tener en cuenta factores operativos y un tiempo más extenso en la implementación de la práctica de Mindfulness, con la posibilidad de hacer mediciones a otras variables psicológicas relacionadas con las habilidades para la vida; de igual forma, considerar la variable del desempeño académico y cognitivo, que en la presente investigación no se adoptó, pero es un factor importante para tener en cuenta.

Es importante, concienciar en las dinámicas entre docentes y estudiantes, puesto que en la medida que haya una mejor sinergia se puede prevenir estrés escolar y por ende problemáticas a partir de ello. Una mejor relación y comunicación puede apuntar al logro de mejores rendimientos académicos. 
Las técnicas de Mindfulness, hoy día poseen el aval científico necesario para que el sistema educativo plantee seriamente su inclusión como un recurso psicológico dirigido no solo a los estudiantes, sino a docentes y directivos, apuntando así a mejorar el desempeño personal, emocional, cognitivo y académico.

Finalmente, aunque los resultados del presente estudio han sido positivos, hay que ser prudentes respecto a la generalización de los mismos, ya que la muestra de la investigación fue baja, por lo que puede presentar sesgos. Además, es necesario confirmar la permanencia, a lo largo del tiempo, de los resultados obtenidos mediante la realización de medidas de seguimiento que confirmen si dichas mejoras en la empatía se mantienen a largo plazo. De igual forma, es importante considerar que (primero) la implementación de las prácticas de contemplación, como el Mindfulness, deben ser guiadas por profesionales y/o expertos en el tema y (segundo) tener conciencia que la raíz de Mindfulness es el Budismo.

\section{Referencias bibliográficas}

Aguilar, G., \& Musso, A. (2008). La meditación como proceso cognitivo conductual. Suma Psicológica, 15, 241-258.

Alajmo, T., \& Jiménez, L. (2013). Evaluación del efecto de un programa basado en mindfulness para el manejo de ansiedad en niños escolarizados. Universidad de la Sabana. Instituto de Postgrados - FORUM Facultad de Psicología. Especialización en Psicología Clínica de la Niñez y la Adolescencia.

Arden, J. (2002). Surviving job stress: How to overcome workday pressures. Franklin Lakes. New York: Career Press Incorporated.

Arguís, R., Bolsas, A., Hernández, S., \& Salvador, M. (2012). Programa "Aulas felices" Psicología positiva aplicada a la educación. Equipo SATI. Zaragoza, España.

Austin, J. H. (1998). Zen and the Brain. Cambridge: MIT Press.

Avenanti, A., Minio-Paluello, I., Bufalari, I., \& Aglioti, SM. (2006). Stimulusdriven modulation of motor-evoked potentials during observation of others' pain. Neuroimage. 32. 316-24.

Baer, R. (2003). Mindfulness Training as a Clinical Intervention: A Conceptual and Empirical Review. Clinical Psychology: Science and Practice. 10, (2), 125-143. 
Barraza, A. (2005) Característica del estrés académico de los alumnos de educación media superior. INED, (4), 15-20. Universidad pedagógica de Durango

Bishop, S. R., et al. (2004). Mindfulness: A proposed operational definition. Clinical Psychology: Science and Practice, 11, 230-241.

Bögels, S. M., et al. (2008). Mindfulness training for adolescents with externalizing disorders and their parents. Behavioral and Cognitive Psychotherapy, 36, 193-209.

Botvinick M, Jha AP, Bylsma LM, Fabian SA, Solomon PE, Prkachin KM. (2005). Viewing facial expressions of pain engages cortical areas involved in the direct experience of pain. Neuroimage, 25, 312-9.

Buela-Casal, G., Fernández, L., \& Carrasco, T. (1997) Psicología Preventiva. Madrid: Editorial Pirámide.

Campagne, D. M. (2004). "Teoría y fisiología de la meditación". Cuadernos de Medicina Psicosomática y Psiquiatría de Enlace, 69/70, 15-30.

Carr, L., et al (2003). Neural mechanisms of empathy in humans: a relay from neural systems for imitation to limbic areas. Proc Natl Acad Sci USA, 100, 5497-502.

De Paúl, J. (2003). Empatía y maltrato físico. En Intervención Psicosocial, 2002, 11 º 1, 57-69.

Dumont, M., LeClerc, D., \& Deslandes, R. (2003). Ressources personnelles et detresse psychologique en lien avec le rendement scolaire et le stress chez des eleves de quatrieme secondaire. / Personal resources and psychological distress in association with the school performance and stress of fourth secondary students. Canadian Journal of Behavioural Science, 35 (4), 254-267.

Dunn, B. R., Hartigan, J. A., \& y Mikulas, W. L. (1999). “Concentration and mindfulness meditations: Unique forms of consciousness?" Applied Psychophysiology and Biofeedback, 24, 147165.

Eisenberg, N. (2000). Emotion, regulation, and moral development. Annu Rev Psychol, 51, 665697.

Ekman, P., \& Gyatso, T. (2009). Sabiduría emocional. Barcelona. Editorial: Kairós S.A.

Feshbach, N., \& Feshbach, S. (1982) Empaty training and the regulatión of aggession. Potentialities and limitations, pp. 599-413.

Forero, J., López, H., \& Pardo, N. (2004). Factores psicosociales asociados al estrés estudiantil de un colegio privado de ciudad de Panamá. Universidad de La Sabana.

Franco, C. (2007). Técnicas de relajación y desarrollo personal. Granada: Cepa. 
Franco, C., Soriano, E., \& Justo, E. (2010). Incidencia de un programa psicoeducativo de mindfulness (conciencia plena) sobre el autoconcepto y rendimiento académico de estudiantes inmigrantes sudamericanos residentes en España. Revista Iberoamericana de Educación. No. 53/6.

García, L. et al. (2011). Intimidación entre iguales (bullying): empatía e inadaptación social en participantes de bullying. Revista de investigación en Psicología, 14 (2), 271 - 276.

González, A., Lemus, S., \& Leal, P. (2002). Proyecto de grado, Factores psicosociales asociados al estrés en estudiantes de bachillerato y primaria de siete colegios oficiales del municipio de Chía, Universidad de La Sabana.

Holen, A. (2006). Inner Strength: free mental attitude in Acem meditation. Oslo: Acem International.

Lacoboni, M. (1999) Cortical mechanisms of human imitation. Science, 286, 2526-8.

Lacoboni, M., et al. (2005). Grasping the intentions of others with one's own mirror neuron system. PLoS Biol, 3(79).

Jevning, R. (1988). "Integrated metabolic regulation during states of decreased metabolism, similarity to fasting: A biochemical hypothesis". Physiology and Behavior, 43, 735-737.

Jevning, R., Anand, R., Biedebach, M., \& Fernando, G. (1996). "Effects on region cerebral blood flow of transcendental meditation”. Physiology and Behavior, 59(3), 399-402.

Jiménez, L. (2011) Salud mental en adolescentes y escala de Mindfulness para adolescentes. Recuperado de: http://www.mindfulkidscolombia.blogspot.co m.es/2011/07/salud-mentalen-adolescentes-y-escala.html

Jha, A. P., Krompinger, J., \& Baime, M. J. (2007) Mindfulness training modifies subsystems of attention. Cogn Affect Behav Neurosci. 7(2):109-19. Recuperado en: http://www.ncbi.nlm. nih.gov/pubmed/17672382 jueves, 2 de octubre de 2013.

Kabat-Zinn, J., Lipworth, L., \& Burney, R. (1985). The clinical use of mindfulness meditation for selgulation of chronic pain. Journal of Behavional Medicine, 8, 163-190.

Kabat-Zinn, J. (1990). Full Catastrophe Living: Using the Wisdom of Your Body and Mind to Face Stress, Pain and Illness. New York: Delta.

Kabat-Zinn, J. (2005). Coming to our senses: Healing ourselves and the world throught mindfulness. New York: Hyperion.

Keysers, C., et al. (2004). A touching sight: SII/PV activation during the observation and experience of touch. Neuron, 42, 335-46. 
León, B. (2008). Atención plena y rendimiento académico en estudiantes de enseñanza secundaria. European Journal of Education and Psychology. Vol 1, No 3. pp. 17-26

Martínez, E., \& Díaz, D. (2007). Una aproximación psicosocial al estrés escolar. Educación y Educadores, 10 (2), 11-22.

Martorell, M., González, R., \& Calvo, A. (1998). Cuestionario de Conducta Prosocial. Valencia: Universidad de Valencia.

Morrison, I, Peelen, MV, \& Downing, PE. (2007). The sight of others' pain modulates motor processing in human cingulate cortex. Cereb Cortex, 17, 2214-2222.

Moya-Albiol, L., et al. (2005). Respuesta psicológica de estrés en una jornada laboral. Phicothema, 17, 205-211.

Polo, A., Hernández, J., \& Poza, C. (1996). Evaluación del estrés académico en estudiantes universitarios. Revista Ansiedad y estrés. Recuperado de: http://www.unileon.es/ estudiantes/atencionuniversitario/articulo.pdf

Rey, C. (2003). La medición de la empatía en preadolescentes y adolescentes varones: adaptación y validación de una escala. Revista Latinoamericana de Psicología, 35 (2), 185-194.

Richaud, M. (2008). Evaluación de la empatía en población infantil argentina. Revista IIPSI, 11 (1), 101-115.

Rizzolatti G., Fogassi L., \& Gallese V. (2001). Neurophysiological mechanisms underlying the understanding and imitation of action. Nat Rev Neurosci, 2, 661-70.

Robinson, N. S., Garber, J., \& Hilsman, R. (1995). "Cognitions and stress: Direct and moderating effects on depressive versus externalizing symptoms during the junior high school transition”. Journal of Abnormal Psychology, 104 (3), 453-463.

Rodríguez, G., Roque, D., \& Molerio, P. (2002). Estrés laboral, consideraciones sobre sus características y formas de afrontamiento. Psicología Científica. Recuperado de: http:// www.psicologiacientifica.com/publicaciones/biblioteca/ articulos/ar-gonro01_1.htm

Sánchez, G. (2011). Meditación, Mindfulness y sus efectos biopsicosociales. Revisión de literatura. Revista Electrónica de Psicología Iztacala, 14 (2).

Sandín, B. (2003). El estrés: un análisis basado en el papel de los factores sociales. Revista Internacional de Psicología Clínica y de la Salud, 3 (1), 141-157.

Sampieri, R., Fernández, C., \& Baptista, P. (2006). Metodología de la investigación. México. Editorial McGraw-Hill 
Schonert-Reichl, K., \& Lawlor, M. (2010). The effects of a mindfulness-based education program on pre- and early adolescents' well-being and social and emocional competence. Mindfulness.

Simón, V. (2008). Mindfulness y neurobiología. Revista de psicoterapia, 17, pp. 5-30

Solberg, E. E., et al. (2000). "Stress reactivity to and recovery from a standardised exercise bout: a study of 31 runners practising relaxation techniques". British Journal of Sports Medicine, 34, 268-272.

Tettamanti, M., et al. (2005). Listening to action-related sentences activates fronto-parietal motor circuits. J. Cogn Neurosci, 17, 273-281.

Wicker B. (2003) Both of us disgusted in my insula: the common neural basis of seeing and feeling disgust. Neuron, 40, 655-664. 